\title{
Perception towards live virtual clinical skills session: a pilot study
}

\author{
Hamzah, R., Sam, C.P., Siang, D.T.J. \\ International Medical University, Malaysia
}

\section{Background:}

Virtual learning refers to instruction in a learning environment where educators and students are separated by time or space. Due to this pandemic, all clinical skills teaching sessions have been converted to online, one way of teaching clinical skills was used pre-recorded video. There is a challenge to teach clinical skills online because clinical skills require hands-on experience compared to watch video. Virtual learning could consider as a turning point in medical education during this pandemic. Clinical Skills \& Simulation Centre (CSSC) have conducted the live streaming on clinical skills teaching session. This pilot study aimed to evaluate the perception of Live Virtual Clinical Skills (LVCS) sessions.

\section{Method:}

CSSC have conducted LVCS session for medical students. The simulation session was conducted using B-line recording system. The B-line system captures the teaching sessions and streams via Microsoft TEAM. Students viewed their clinical skills teaching sessions from home while the lecturer demonstrates the skills on a simulated patient through a LIVE demonstration. Data was collected from preclinical and clinical year medical students who have participated in the LVCS session. Questionnaires were distributed to 33 participants.

\section{Results:}

19 participants $(57.5 \%)$ responded to the questionnaire. The questionnaire showed internal consistency of $\alpha=0.935$. The participants expressed that LVCS $(n=14,73.7 \%)$ was preferred when compared to video-assisted learning $(n=5,26.3 \%)$. The mean score for the understanding of the subjects using LVCS was 3.84; enjoyment of LVCS was 3.79; interactive during LVCS was 3.63, and quality of the LVCS was 3.26. The overall mean score of the perception toward the LVCS was 3.63.

\section{Conclusions:}

Based on the results, the LVCS sessions viewed as the preferred method in clinical skills teaching and learning activities as students able to receive real-time feedback. Hence, LVCS teaching session is recommended. LVCS session is a valuable tool to enhance students' learning in the pandemic. LVCS may serve as an appropriate teaching methodology during the COVID-19 pandemic.

Key Words: Live virtual, Clinical skills, Perception 